\title{
Hybrid Filter Based Simultaneous Localization and Mapping for a Mobile Robot
}

\author{
Amir Panah ${ }^{1,2}$ and Karim Faez ${ }^{3}$ \\ ${ }^{1}$ Mechatronics Research Laboratory, Qazvin Islamic Azad University, Qazvin, Iran \\ ${ }^{2}$ Young Researchers Club, Qazvin Islamic Azad University, Qazvin, Iran \\ ${ }^{3}$ Electrical Engineering Department, Amirkabir University of Technology, Tehran, Iran \\ amir.panah@qiau.ac.ir, kfaez@aut.ac.ir
}

\begin{abstract}
A mobile robot autonomously explores the environment by interpreting the scene, building an appropriate map, and localizing itself relative to this map. This paper presents a Hybrid filter based Simultaneous Localization and Mapping (SLAM) approach for a mobile robot to compensate for the Unscented Kalman Filter (UKF) based SLAM errors inherently caused by its linearization process. The proposed Hybrid filter consists of a Multi Layer Perceptron (MLP) for neural network and UKF which is a milestone for SLAM applications. The proposed approach, based on a Hybrid filter, has some advantages in handling a robotic system with nonlinear motions because of the learning property of the MLP neural network. The simulation results show the effectiveness of the proposed algorithm comparing with an UKF based SLAM.
\end{abstract}

Keywords: Hybrid filter SLAM, MLP, SLAM, UKF.

\section{Introduction}

Currently, SLAM which is a relatively new subfield of robotics, is one of the most widely researched major subfields of mobile robotics. In order to solve SLAM problems, statistical approaches, such as Bayesian Filters, have received widespread acceptance [7]. Some of the most popular approaches for SLAM include using a Kalman filter (KF), an extended Kalman filter (EKF) and an unscented Kalman filter (UKF) and a particle filter [8]. The UKF SLAM makes a Gaussian noise assumption for the robot motion and its observation. In addition, the amount of uncertainty in the UKF SLAM algorithm must be relatively small; otherwise, the linearization in the UKF tends to unbearable errors. The UKF uses the unscented transform to linearize the motion and measurement models [13]. MLP neural network, adaptive to environmental information flowing through during the process, can be combined with an UKF to compensate for some of the disadvantages of an UKF SLAM approach [4],[12].

Qi Song and Yuqing He [9] in order to overcome the drawback of the normal unscented Kalman filter a novel adaptive UKF is developed and applied to nonlinear joint estimation of both time-varying states and modeling errors for helicopter. The filter is composed of two parallel master-slave UKFs, while the master UKF estimates the states/parameters and the slave one estimates the diagonal elements of the noise 
covariance matrix for the master UKF. Such a mechanism improves the adaptive ability of the UKF and enlarges its application scope.

Zhi Jun Yu et al [14] a new neural network aided Unscented Kalman filter is presented for tracking maneuvering target in distributed acoustic sensor networks. In this approach that using an offline neural network to correct these errors, the nonlinear inferring process is done by the normal Unscented Kalman filter. This method doesn't need complex modeling for tracking maneuvering target and is very suitable for realtime implementation.

Choi et al [2] solved the SLAM problem with a neural network based on an extended Kalman filter. According to the research results, the EKF SLAM based on Neural Network, shows better performance than the EKF SLAM.

Ronghui Zhan and Jianwei Wan [10] presents a robust learning algorithm for an multilayered neural network based on UKF. Since it gives a more accurate estimate of the link weights, the convergence performance is improved. This algorithm is then extended further to develop a neural network aided UKF for nonlinear state estimation.

In this paper, we present a Hybrid approach using MLP neural network and UKF based SLAM problem for decreasing uncertainty in compare to SLAM using UKF. We also discuss the effectiveness of MLP algorithm to handle nonlinear properties of a mobile robot.

Some related algorithms on SLAM are described in section 2, and the Hybrid SLAM algorithm is presented in section 3. Section 4 shows the simulation results of the SLAM based on UKF, and Hybrid filter. Concluding remarks, discussion and further research are discussed in section 5 .

\section{Related Algorithms for SLAM}

\subsection{Multi Layer Perceptron Neural Network}

Frequently, neural networks are used especially in modeling and simulation of nonlinear systems. Neural networks have two fundamental characteristics of learning based on presentation experimental data and structural parallel. Specially, MLP which was evolved from single layer perceptron with a parallel processing pattern, has been proposed in the early days. MLP is suitable turn out for nonlinear information. The MLP with hidden layers with one or more input and output nodes, is used a typical feedforward neural network model used as a universal approximator. The output signals are generated through the homogeneously nonlinear function after summing signal values for each of the input nodes. In this process, signals are multiplied with appropriate weights and added with some bias values [5],[15].

\subsection{Unscented Kalman Filter}

This filter is built based on transformation as unscented transformation. In the UKF, there is no need to calculate Jacobian matrix. Since, the processing noise in this system is accumulative; therefore the augmented state vector is used to implementation this approach. In this approach, the mean and covariance estimation are calculated with considering the second order of the Taylor series [6]. 


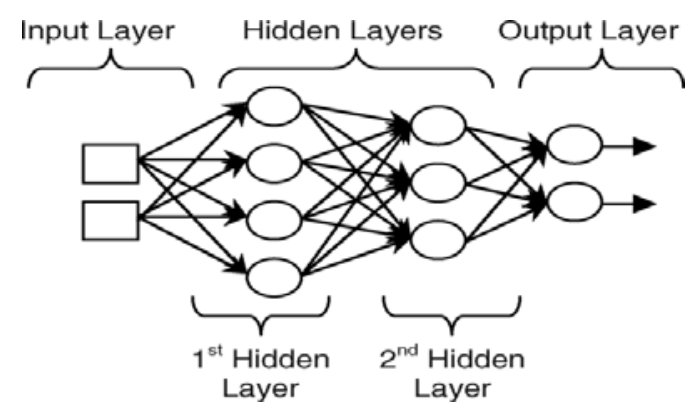

Fig. 1. A Multi Layer Perceptron Neural Network Structure

Suppose that a random variable $x$ with mean $\mu$ and covariance $P_{X}$ is given and also a random variable $z$ related with $x$ using: $z=f(x)$. Calculation Problem of the mean and covariance of $\mathrm{z}$ is the same as the predicted and corrected problem in UKF stages for a nonlinear system. In the Unscented Transformation method, a set of weighted points called sigma points are used to reach the mean and the covariance of random variable $z$. This sigma points should be selected in a way that have enjoy the mean $\mu$ and covariance $P_{X}$. For n-dimensional random variable with mean $\mu$ and covariance $P_{X}, 2 \mathrm{n}+1$ instance points are selected as follows. $(0<\mathrm{i}<\mathrm{n})$

$$
\begin{gathered}
X_{0}=\mu \quad, \quad W_{0}=\frac{\lambda}{n+\lambda} \\
X_{i}=\mu+\left(\sqrt{(n+\lambda) P_{x}}\right)_{i} \quad, \quad W_{i}=\frac{\lambda}{2(n+\lambda)} \\
X_{i+n}=\mu-\left(\sqrt{(n+\lambda) P_{x}}\right)_{i} \quad, \quad W_{i+n}=\frac{\lambda}{2(n+\lambda)} \\
\lambda=\alpha^{2}(n+\beta)-n
\end{gathered}
$$

$\mathrm{N}$ is the number of augment state. $\alpha$ and $\beta$ are the coefficients that the estimation error can be minimized by adjusting them, and also their values influence on the error rate resulted from the higher terms in Taylor series. In the above mentioned equations, $k \in R$ and $\left(\sqrt{\left.(n+\lambda) P_{x}\right)}\right.$, the i-th row or column of the matrix is the square root of $(n+\lambda) P_{x}, W_{i}$ is the weight belongs to each point and $\mathrm{k}$ also is used for more accurate adjusting of UKF [6]. According to Unscented Transformation algorithm, each point in a set of points is first mapped to a new point by a nonlinear function, which results in a new set of sigma points. Then, we calculate the mean and the covariance values of the new random variable. Consider the following nonlinear system.

$$
\begin{gathered}
x_{k}=f\left(x_{k-1}, u_{k-1}, \varepsilon_{k}\right) \\
z_{k}=h\left(x_{k}, u_{k}, \delta_{k}\right)
\end{gathered}
$$

Where $x$ is the state vector and $u$ is control input, $\mathcal{E}$ is the system noise and $\delta$ is the measurement noise. In the first phase of implementing this filter, the augment state vector is formed as follows. 


$$
X_{k}^{a}=\left[\begin{array}{c}
X_{k} \\
\varepsilon \\
\delta
\end{array}\right]
$$

In the following, we will have all formulas used in the UKF which include two main sections, the Measurement Update and the Time Update [11].

- The Time Update

$$
\begin{gathered}
X_{k}^{a}=f\left(X_{k}^{a}, u_{k}, \varepsilon_{k}\right) \\
\mu_{k}=\sum_{i=0}^{2 n} w_{i} X_{i, k}^{a} \\
P_{k}=\sum_{i=0}^{2 n} w_{i}\left[X_{i, k}^{a}-\mu_{k}\right]\left[X_{i, k}^{a}-\mu_{k}\right]^{T} \\
z_{k}=h\left(x_{k}, u_{k}, \delta_{k}\right) \\
\bar{z}_{k}=\sum_{i=0}^{2 n} w_{i} z_{k}
\end{gathered}
$$

- $\quad$ The Measurement Update

$$
\begin{gathered}
P_{\mathrm{x}_{\mathrm{k}} \mathrm{x}_{\mathrm{k}}}=\sum_{i=0}^{2 n} w_{i}\left[z_{i, k}-\bar{z}_{k}\right]\left[z_{i, k}-\bar{z}_{k}\right]^{T} \\
P_{x_{k} y_{k}}=\sum_{i=0}^{2 n} w_{i}\left[X_{i, k}^{a}-\mu_{k}\right]\left[z_{i, k}-\bar{z}_{k}\right]^{T} \\
K_{k}=P_{x_{k} y_{k}} P_{x_{k} x_{k}}{ }^{-1} \\
\mu_{k}=\mu_{k}+K_{k}\left(z_{k}-\bar{z}_{k}\right) \\
P_{k}=P_{k}-K_{k} P_{x_{k} x_{k}} K_{k}^{T}
\end{gathered}
$$

Where $X_{k}^{a}, \mu_{k}, P_{k}, Z_{k}, \bar{Z}_{k}, P_{x_{k} x_{k}}, P_{x_{k} y_{k}}$ and $K_{k}$, are defined as motion model, predicted mean, observation model, predicted observation, innovation covariance, cross correlation matrix and Kalman gain, respectively.

\section{SLAM Algorithm Using Hybrid Filter}

A new Hybrid filter SLAM using an UKF and a MLP is proposed here. The mean $\boldsymbol{u}_{k}$ which is derived from environmental information values $(x y \theta \varepsilon \delta)$ using the MLP algorithm, is entered to the prediction step, as shown in Fig. 2. 


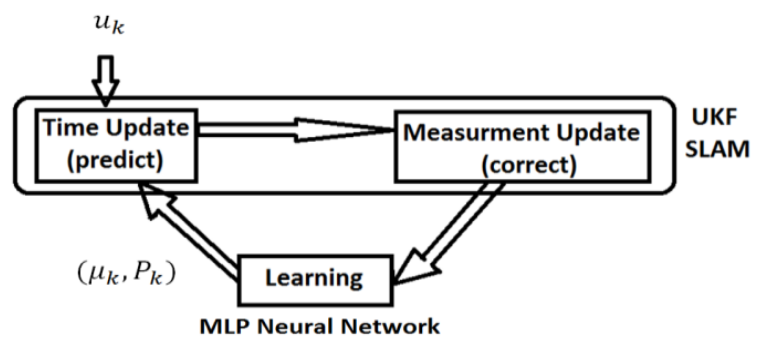

Fig. 2. The architecture of the Hybrid filter SLAM

In this paper, Basic inputs are mean, covariance which are calculated by prior input, $\boldsymbol{u}_{k-1}$, and present input, $\boldsymbol{u}_{k}$. The robot calculates the prior mean and covariance in The Time Update step and then in The Measurement Update step, calculates a Kalman gain, present mean and covariance and defined features.

\subsection{Time Update (Predict)}

In the following article, The Hybrid filter SLAM algorithm is described using a robot's pose and features, such as the location of landmarks. For the SLAM, the basic motion model of the mobile robot needs to be presented in the fallowing. A configuration of the robot with a state equation $X^{a}=(x y \theta \varepsilon \delta)^{T}$, has the form of Eq. (18).

$$
\begin{gathered}
X_{k}^{a}=\left[\begin{array}{l}
x_{k} \\
y_{k} \\
\theta_{k} \\
\varepsilon_{k} \\
\delta_{k}
\end{array}\right]=\left[\begin{array}{c}
x_{k-1}+v_{k} \Delta t \cos \left(\theta_{k}\right) \\
y_{k-1}+v_{k} \Delta t \sin \left(\theta_{k}\right) \\
\theta_{k-1+} v_{k} \Delta t \sin \left(\frac{\Delta \theta}{L}\right) \\
\varepsilon_{k-1} \\
\delta_{k-1}
\end{array}\right] \\
u_{k}=v_{k}+N\left(0, M_{k}\right)
\end{gathered}
$$

Where $v_{k}$ is velocity of wheels, and $\mathrm{L}$ is the width between the robot's wheels, and $\Delta t$ is the sampling period. Finally, $M_{k}$ describes the covariance matrix of the noise in control space. The state equation for landmarks, is combined with the robot position, is denoted by the vector $Y_{k}$, where $\mathrm{c}$ is the number of landmarks. $(0<\mathrm{i}<\mathrm{c})$

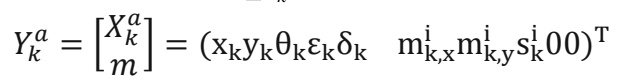

The state transition probability of a Hybrid filter SLAM has the form of Eq. (21).

$$
X_{k}^{a}=f\left(X_{k-1}^{a}, u_{k-1}\right)+N\left(0, \varepsilon_{k}\right)
$$

Under the linearity assumption where $f$ represents the nonlinear functions, $\boldsymbol{\varepsilon}_{k}$ is the process noise, and $\boldsymbol{u}_{k}$ is control input. For the Taylor expansion of function, $f$ its partial derivative is used with respect to $X_{k}^{a}$, as shown in Eq. (22). 


$$
f^{\prime}\left(X_{k-1}^{a}, u_{k}\right)=\frac{\partial f\left(X_{k-1}^{a}, u_{k}\right)}{\partial X_{k}^{a}}
$$

The continuing linearization using of $f$ is approximated at $\boldsymbol{U}_{k}$ and $\boldsymbol{u}_{k-1}$ as shown in Eq. (23).

$$
f\left(X_{k-1}^{a}, u_{k}\right)=f\left(\mu_{k-1}, u_{k}\right)+f^{\prime}\left(\mu_{k-1}, u_{k}\right)\left(X_{k}^{a}-\mu_{k-1}\right)
$$

With the replacement values obtained from equations $1,2,3,4,18$, prior mean and covariance have the following form of:

$$
\begin{gathered}
\mu_{k}=\sum_{i=0}^{2 n} w_{i} X_{i, k}^{a} \\
P_{k}=\sum_{i=0}^{2 n} w_{i}\left[X_{i, k}^{a}-\mu_{k}\right]\left[X_{i, k}^{a}-\mu_{k}\right]^{T} \\
z_{k}=\left[\begin{array}{c}
\sqrt{\left(m_{k, x}^{i}-x_{k}\right)^{2}+\left(m_{k, y}^{i}-y_{k}\right)^{2}} \\
\tan ^{-1}\left(\frac{m_{k, y}^{i}-y_{k}}{m_{k, x}^{i}-x_{k}}\right)-\theta_{k}
\end{array}\right]+N\left(0, \delta_{k}\right) \\
m^{i}=\left(m_{x}^{i} m_{y}^{i}\right)^{T} \\
\bar{z}_{k}=\sum_{i=0}^{2 n} w_{i} z_{k}
\end{gathered}
$$

\subsection{The Measurement Update (Correct)}

To obtain the Kalman gain $K_{k}$, we need to calculate $P_{x_{k} x_{k}}$ and $P_{x_{k} y_{k}}$ in the feature based maps. To obtain the values $P_{x_{k} x_{k}}$ and $P_{x_{k} y_{k}}$, it is necessary to calculate $X_{k}^{a}, \mu_{k}$, $Z_{k}, \bar{z}_{k}$ that are calculated in equations $18,24,26,28$, with replacement of these values, we will have the following equations.

$$
\begin{gathered}
P_{x_{k} x_{k}}=\sum_{i=0}^{2 n} w_{i}\left[z_{i, k}-\bar{z}_{k}\right]\left[z_{i, k}-\bar{z}_{k}\right]^{T} \\
P_{x_{k} y_{k}}=\sum_{i=0}^{2 n} w_{i}\left[X_{i, k}^{a}-\mu_{k}\right]\left[z_{i, k}-\bar{z}_{k}\right]^{T} \\
K_{k}=P_{x_{k} y_{k}} P_{x_{k} x_{k}}{ }^{-1}
\end{gathered}
$$

In the following, complete combined MLP algorithm with UKF is described to SLAM of the mobile robot. MLP are involved with train through input data and measurement values. In the training process, weights are decided based on the relation of input data and each hidden layers. MLP Neural Network needs higher weight to objective value on the higher relations between poses and heading angle with comparing to measurement. 
To apply a MLP, the mean values for each element are divided, and substituted by inputs of the MLP algorithm for each mean value. This research utilizes the MLP with two hidden layers, so the process equation is derived as Eq. (32). Under the assumption that this process does not have any bias, the $n, n_{1}, n_{2}$ and $n_{3}$ describe the number of input nodes, the first hidden layer's nodes, the second hidden layer's nodes and output layer's nodes with A, B and C, the number of nodes, respectively [8].

$$
\begin{aligned}
\hat{\mu}_{k}^{n}=\xi\left[\sum_{n_{3}=0}^{C-1} w_{k}^{n_{2} n_{3}} \varphi_{k}^{n_{2}}\right]=\xi\left[\sum_{\gamma=0}^{C-1} w_{k}^{n_{2} \gamma} \xi\left[\sum_{B=0}^{B-1} w_{k}^{n_{1} n_{2}} \varphi_{k}^{n_{1}}\right]\right] \\
=\xi\left[\sum_{n_{3}=0}^{C-1} w_{k}^{n_{2} n_{3}} \xi\left[\sum_{n_{2}=0}^{B-1} w_{k}^{n_{1} n_{2}} \xi\left[\sum_{n_{1}=0}^{A-1} w_{k}^{n n_{1}} \bar{\mu}_{k}^{n}\right]\right]\right] \\
\left(0 \leq n_{1} \leq A-1,0 \leq n_{2} \leq B-1,0 \leq n_{3} \leq C-1\right)
\end{aligned}
$$

The next process to obtain the prior mean and the covariance is to update the results from Eq. (32). The process described in the above 5 steps repeats until the end of the navigation.

$$
\begin{gathered}
\mu_{k}=\hat{\mu}_{k}+K_{k}\left(z_{k}-\bar{z}_{k}\right) \\
P_{k}=P_{k}-K_{k} P_{x_{k} x_{k}} K_{k}{ }^{T}
\end{gathered}
$$

\section{Simulations}

To show the effectiveness of the proposed algorithm, the Matlab code, developed by Bailey [1], was modified. The simulation was performed with constraints on velocity, steering angle, system noise, observation noise, etc., for a robot with a wheel diameter of $1[\mathrm{~m}]$, maximum speeds of $3[\mathrm{~m} / \mathrm{sec}]$, maximum steering angle and speed are $25\left[^{\circ}\right]$ and $15[\% \mathrm{sec}]$, respectively. The control input noise is assumed to be a zero mean Gaussian with $\sigma_{v}(=0.2[\mathrm{~m} / \mathrm{s}])$ and $\sigma_{\varphi}\left(=3\left[^{\circ}\right]\right)$. For observation, the number of arbitrary features around waypoints was used. In the observation step, a range bearing sensor model and an observation model were used to measure the feature position and robot pose, which includes a noise with level of $0.1[\mathrm{~m}]$ in range and $1\left[^{\circ}\right]$ in bearing. The sensor range is restricted to $15[\mathrm{~m}]$.In this research, a rectangular navigation case of the robot are surveyed. Specifications of navigation map are described in Table1.

Table 1. Fundamental specification for navigation

\begin{tabular}{|c|c|}
\hline Item & Rectangular \\
\hline Feature & 40 \\
\hline Waypoint & 5 \\
\hline Area[m] & $30 * 30$ \\
\hline
\end{tabular}




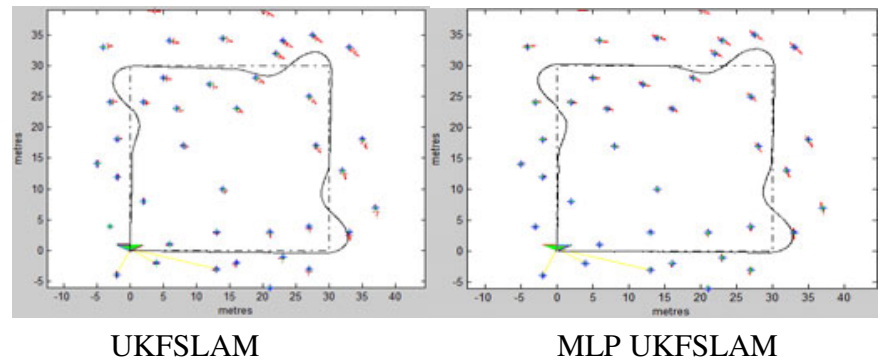

Fig. 3. Navigation result on rectangular map

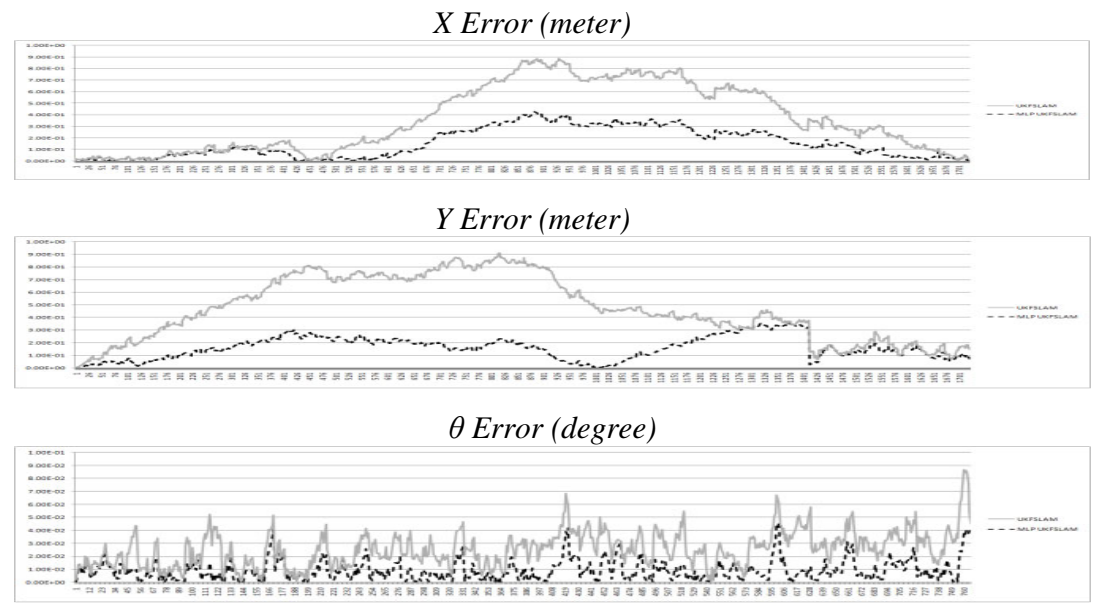

Fig. 4. Navigation errors on rectangular map

\subsection{Navigation on Rectangular Map}

In the case of rectangular navigation, the UKF based on navigation and Hybrid filter based on navigation are shown in Fig. 3. The dashed line, show the paths of robots should traverse and the bold black line is Robot path, based on data described by the actual odometry. In Fig. 4, the gray bold line and the dashed black line are the $\mathrm{x}, \mathrm{y}$, and $\theta$ errors in the case of UKF SLAM and Hybrid filter SLAM, respectively.

\section{Conclusions}

The SLAM since the robot keeps track of its location by maintaining a map of the physical environment and an estimate of its position on that map, is one of the most fundamental problems in the quest for autonomous mobile robots. This paper 
proposes UKF SLAM based on MLP method for a mobile robot, to make up for the UKF SLAM error inherently caused by its linearization process and noise assumption. The proposed algorithm consists of two steps: the MLP Neural Network and the UKF algorithm. The simulation results show that the efficiency of the proposed algorithm based on MLP as compared with the UKF SLAM. To verify the effectiveness of the proposed algorithm, simulation in Matlab shown UKF SLAM has more errors than Hybrid filter SLAM. In addition, the simulation results confirm the Hybrid filter SLAM is more stable for robot navigation. In the future Research up on the robustness of the proposed algorithm, will verify under harsh and real-time condition using of fuzzy logic or structure change of neural network.

\section{References}

1. Bailey, T.: http: / / www . personal .acfr.usyd.edu.au/tbailey

2. Choi, M.Y., Sakthivel, R., Chung, W.K.: Neural network aided extended Kalman filter for SLAM problem. In: IEEE International Conference on Robotics and Automation, pp. 1686-1690 (2007)

3. Cho, S.H.: Trajectory Tracking Control of a Pneumatic X-Y Tabel using Neural Network Based PID Control. Int. J. Precis. Eng. Manuf. 10(5), 37-44 (2009)

4. Harb, M., Abielmona, R., Naji, K., Petriul, E.: Neural networks for environmental recognition and navigation of a mobile robot. In: IEEE International Instrumentation and Measurement Technology Conference, pp. 1123-1128 (2008)

5. Hu, Y.H., Hwang, J.N.: Handbook of Neural Network Signal Processing, pp. 3.1-3.23. CRC Press, Boca Raton (2001)

6. Julier, S.J., Uhlmann, J.K.: A New Extension of Kalman Filter to Nonlinear Systems. In: Proceedings of AeroSense: The 11th Int. Symp. on Aerospace/Defence Sensing, Simulation and Contro. (1997)

7. Kim, J.M., Kim, Y.T., Kim, S.S.: An accurate localization for mobile robot using extended Kalman filter and sensor fusion. In: IEEE International Joint Conference on Neural Networks, pp. 2928-2933 (2008)

8. Choi, K.-S., Lee, S.-G.: Enhanced SLAM for a Mobile Robot using Extended Kalman Filter and Neural Networks. International Journal Of Precision Engineering And Manufacturing 11(2), 255-264 (2010)

9. Song, Q., He, Y.: Adaptive Unscented Kalman Filter for Estimation of Modelling Errors for Helicopter. In: 2009 IEEE International Conference on Robotics and Biomimetics, Guilin, China, December 19-23, 2009, pp. 2463-2467 (2009)

10. Zhan, R., Wan, J.: Neural Network-Aided Adaptive Unscented Kalman Filter for Nonlinear State Estimation. IEEE Signal Processing Letters 13(7), 445-448 (2006)

11. Page, F.S.: Multiple-Object sensor Management and optimization. PHD thesis, in the faculty of Engineering, Science and Mathematics School of Electronics and Computer science (June 2009)

12. Vafaeesefat, A.: Optimum Creep Feed Grinding Process Conditions for Rene 80 Supper Alloy Using Neural network. Int. J. Precis. Eng. Manuf. 10(3), 5-11 (2009)

13. Zhu, J., Zheng, N., Yuan, Z., Zhang, Q., Zhang, X.: Unscented SLAM with conditional iterations. In: 2009 IEEE Intelligent Vehicles Symposium, pp. 134-139 (2009) 
14. Yu, Z.-J., Dong, S.-L., Wei, J.-M., Xing, T., Liu, H.-T.: Neural Network Aided Unscented Kalman Filter for Maneuvering Target Tracking in Distributed Acoustic Sensor Networks. In: International Conference on Computing: Theory and Applications, Kolkata, India, March 5-7 (2007)

15. Zu, L., Wang, H.K., Yue, F.: Artificial neural networks for mobile robot acquiring heading angle. In: Proceedings of the Third International Conference on Machine Learning and Cybernetics, pp. 26-29 (2004) 